\title{
Microsatellite Genetic Markers of Saccharrum spp., and Erianthus sp. on Their Hybrids
}

\author{
Mala Murianingrum ${ }^{1,2}$, Taryono ${ }^{1,3 *}$ and Rani Agustina Wulandari ${ }^{1}$ \\ ${ }^{1}$ Faculty of Agriculture, Gadjah Mada University, Bulaksumur, Yogyakarta, Indonesia \\ Jln. Flora no. 1, Bulaksumur, Sleman, Yogyakarta, Indonesia \\ ${ }^{2}$ Indonesian Sweetener and Fiber Crops Research Institute, Malang, East Java, Indonesia \\ Jln. Raya Karangploso Km. 4., Malang, East Jawa, Indonesia \\ ${ }^{3}$ Agrotechnology Innovation Centre, Universitas Gadjah Mada, Yogyakarta, Indonesia \\ Jl. Tanjung Tirto, Kali Tirto, Berbah, Tanjung, Kalitirto, Berbah, Kabupaten Sleman, Yogyakarta \\ *Corresponding author's email: tariono60@gmail.com
}

Received: 12 $2^{\text {nd }}$ April 2018; Revised: $15^{\text {th }}$ October 2018; Accepted: $15^{\text {th }}$ October 2018

\begin{abstract}
Progeny identification is the important step that should be done after hybridization. However, polyploidy, aneuploidy and the high chromosome segregation in sugarcane which results various phenotypic characteristics variation and environmental effects become limiting factors to identify the progenies based on morphological characteristic. Microsatellite as one of molecular marker which has codominance inheritance, multiallelic, abundant in the genome and does not influenced by environmental factor is the best tool to asses the crossing fidelity accurately. This research aimed to identify the possibility of genetic marker of Saccharum spp. and Erianthus sp. on their hybrid using microsatellite molecular marker. This study was carried out in Molecular Genetic laboratory, Indonesian Sweetener and Fiber Crops Research Institute (ISFCRI) Malang, from August 2016 to July 2017. Eighty-six (86) F1 intraspecific and interspecific progeny, three commercial sugarcane varieties (PSJT941, PS881 and VMC7616) and two wild types (S. spontaneum dan Erianthus sp.) were assessed genetically by three microsatellite markers. Identification of microsatellite genetic markers was conducted by comparing the visualization band results from electrophoresis of each male and female parent through their progenies. All primers could identify Saccharum spp. and Erianthus sp. genetic markers. There were one to eleven Saccharum spp. and Erianthus sp. genetic markers could be identified such as 2-11 PS881-specific alleles; 2-3 VMC7616-specific alleles; 1-5 PSJT941-specific alleles; two S. spontaneum-specific alleles and 1-2 Erianthus-specific alleles. These findings could be used as the advance genetic marker of microsatellite in sugarcane breeding to asses the cross fidelity.
\end{abstract}

Keywords: Erianthus sp., genetic marker, microsatellite, PSJT941, PS881, Sugarcane, VMC7616, S. spontaneum

\section{INTRODUCTION}

Since 1888, conventional sugarcane breeding program through intraspecific and interspecific hybridization had been done. For almost 128 years it has successfully improved important agronomic characteristics such as yield enhancement (productivity) and resistance to biotic and abiotic stresses. In recent years, the purposes of sugarcane breeding programs not only produced sugars but also started to broad utilities such as producing biomass and bioethanol. The genetic improvement has been giving the great contributions to increase the sugarcane productivity until 50\% (Barbosa, et al., 2012), however, there are still many obstacles in conventional sugarcane breeding program.

Sugarcane as a cross-pollinated plant is heterozygous and polyploid, therefore predicting the crossing result become complicated task. Sugarcane and its wild relatives have phenotypic similarity, causing difficulties to identify the progenies based on their morphological characteristics (Wang et al., 2009). Event though both parents have different characteristics, polyploidy, aneuploidy and high chromosome segregation will generate progenies with high variations. Furthermore, not all sugarcane hybrids have high vigorous performances. In addition, many selfed plants has a good vigor (McIntray and Tew 2001). Sugarcane 
breeding also needs great amount of resources and long time process. It takes $12-14$ years to release new varieties (Chandra et al., 2014). Generally, less than one variety to be released in a year with the yield increasing rate $<1 \%$ (Hogarth, 1976 in Tew and Pan, 2010).

DNA molecular marker could be an alternative tool to identify the hybrid because of its advantageous characteristics, such as it is abundant in the genome and not influenced by environmental factor. Microsatellite as one of DNA molecular marker could be the best tool to identify the hybrid due to its co-dominance inheritance, multialelic, widely and scattered in the plant genome. Progenies identification become the most important thing that should be done because it will determine the next step of sugarcane breeding program. Additionally, it is also an imperative task to guarantee the purity of variety identity. Inaccuracy in determining the crossing will affect the next breeding program. It will influence the average of breeding value and finally will refract the decision that should have been taking.

Determination of male and female specific alleles is the first step that should be completed before the assessment of crossing fidelity (authentication) using DNA marker (Tew and Pan, 2010; Costa et al., 2014; Xavier et al., 2014; You et al., 2013; Fliho et al., 2010; Aitken et al., 2007; Pan, 2006). Xavier et al. (2014) using 10 microsatellite primers and had succeed identifying 113.25 (73\%) in average male specific alleles in progenies from polycross. However, Tew and Pan (2010) reported there were 79-99\% male specific alleles that could be identified among the progenies from polycross by microsatellite marker. Alongside with that, using microsatellite marker, Melloni et al. (2014) could identify 28 male specific alleles in progenies derived from polycros of four sugarcane cultivar. Lu et al. (2015) could identify three to six specifik alleles according to pollen and S1 population of sugarcane variety (LCP 85-384) using five microsatellite markers. This study aimed to identify microsatellite genetic marker of Saccharrum spp., and Erianthus sp. on their hybrid.

\section{MATERIALS AND METHODS}

Eighty six (86) F1 progenies from the following crossing were analyzed : six genotypes from PS 881 x PSJT $9451(\mathrm{G}), 25$ genotypes from PS 881 X VMC 76-16 (H), 11 genotypes from PSJT 941 X PS 881 (B), seven genotypes from PSJT 941 X VMC 76-16 (D2), eight genotypes from VMC 76-16 X PS 881 (E2), four genotypes from VMC 76-16 X PSJT 941
(C1), four genotypes from PS 881 X S. spontaneum (C2), three genotypes from PSJT 941 X S. spontaneum (F2), 11 genotypes from VMC 76-16 X S. spontaneum (A), one genotype from PS 881 X Erianthus sp. (E1), four genotypes from PSJT 941 X Erianthus sp. (F1) and two genotypes from VMC 76-16 X Erianthus sp. (D1). The study was conducted in Molecular Genetic laboratory, Indonesian Sweetener and Fiber Crops Research Institute (ISFCRI) Malang, from August 2016 to July 2017. Crossing was conducted in Karangploso experimental garden of Indonesian Sweetener and Fiber Crops Research Institute (ISFCRI) Malang, from January to December 2014.

Crossing was done by marcotting method. The stalks that were consisted of female and male inflorescence were selected randomly from the field. They were cut and then positioned together inside the lantern with male inflorescence was placed above the female inflorescence. Stalks were cut from the field at a stage just prior to when stigma emerged from the tip of panicle which was marked by the appearance of the flag leaves. The stalks were placed in buckets of Hawaiian preserving solution (Heinz and Tew, 1987) during crossing and seed setting which was changed weekly. Pollen test was conducted before crossing. Pollen grains were separately collected and observed microscopically after staining in iodine solution and emasculated by placed the inflorescence in 70\% Alchohol for 5 minutes to eliminate pollen viability at female parent. Furthermore, the progeny of each cross had been selected by their phenotypic appearance (as like as both of parent) for agronomic type only.

DNA was isolated from the tissue of young leaf roll using GeneAll exgene Plant SV mini kit (General biosystem, Korea), following manufacture's intruction. Quality of the DNA was checked on $0.5 \%$ agarose gels in $70 \mathrm{ml}$ TBE $0,5 \mathrm{x}$ and electrophoresis at $100 \mathrm{~V}$ for $30 \mathrm{~min}$.

Three primers had been selected on the basis of showing polymorphism among five parents manually by visualization of different band size differences. This differences were then used as male and female parent genetic marker in the progeny. The three primers were mSSCIR43 (F : ATT CAA CGA TTT TCA CGA G; R : AAC CTA GCA ATT TAC AAG AG), mSSCIR66 (F : AGG TGA TTT AGC AGC ATA; R : CAC AAA TAA ACC CAA TGA) and SMC119CG C (F : TTC ATC TCT AGC CTA CCC CAA; R : AGC AGC CAT TTA CCC AGG A). These microsatellite markers were based on Pan, (2006) research. All microsatellite fragments were 
scored manually for the presence (1) or absence (0) of a band across the 86 progeny and five parents. In addition, the primer could also detect eliminated alleles of parents and additional alleles. Additional alleles are the new alleles that were detected in progeny but did not detected in parents. It could be as a new inter or intrageneric recombination.

PCR final reaction volume was $25 \mu \mathrm{L}$ containing $1 \mu \mathrm{L}$ template DNA; $1 \mu \mathrm{L}$ of each primer (forward dan reverse); 9,5 $\mu \mathrm{L}$ dh2o and 1 unit GoTaq ${ }^{\circledR}$ Green Master Mix Promega Corporation USA. PCR reaction was conducted on a PCR machine Sensoquest Lab Cycler Germany under the program of $940 \mathrm{C}$ for 5 $\mathrm{min}$, followed by 35 cycles of denaturation (940C for $1 \mathrm{~min}$ ), annealing ( $2 \mathrm{~min}$ at $45.50 \mathrm{C}$ for mSSCIR43, 41.30C for mSSCIR66 and 63.50C for SMC119CG), extension $\left(1 \mathrm{~min}\right.$ at $\left.72^{\circ} \mathrm{C}\right)$ and final extension $\left(72^{\circ} \mathrm{C}\right.$ for $5 \mathrm{~min})$. Amplification products were separated by electrophoresis on $2 \%$ gel agarose $(75 \%$

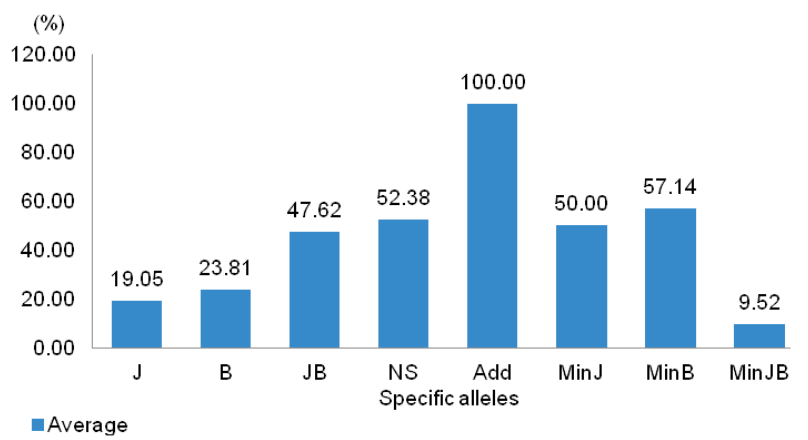

Figure 1. The ability of three primer to detect specific alleles of parents in F1. J: male specific alleles; B: female specific alleles; JB: male and female specific alleles present at different location; NS: male and female alleles present at the same location; Add: additional alleles; MinJ: eliminated male alleles; MinB: eliminated female alleles; MinJB: eliminated male \& female alleles
Metaphore and 25\% agarose) in $70 \mathrm{ml} 1 \mathrm{x}$ TBE buffer and GelRed ${ }^{\mathrm{TM}}$ Nucleic Acid Stain Biotium with US Patents at $100 \mathrm{~V}$ for $180 \mathrm{~min}$. After electrophoresis, the microsatellite products were visualized under UV transilluminator and documented using Geldoc Wealtec KETA (Wealtec Corp).

\section{RESULTS AND DISCUSSION}

\section{Results}

All primers could detect 159 loci but only 60 loci $(37.7 \%)$ that indicated as male and female genetic marker from all crossing. In general, primers had the better ability $(\geq 50 \%)$ to detect common alleles (NS, both of male and female specific alleles present at the same bp), additional alleles (Add), eliminated male (MinJ) and female alleles (MinB) respectively.

Additionaly, primers also had better ability to detect male and female specific alleles in collectively (JB, male and female specific alleles present at different

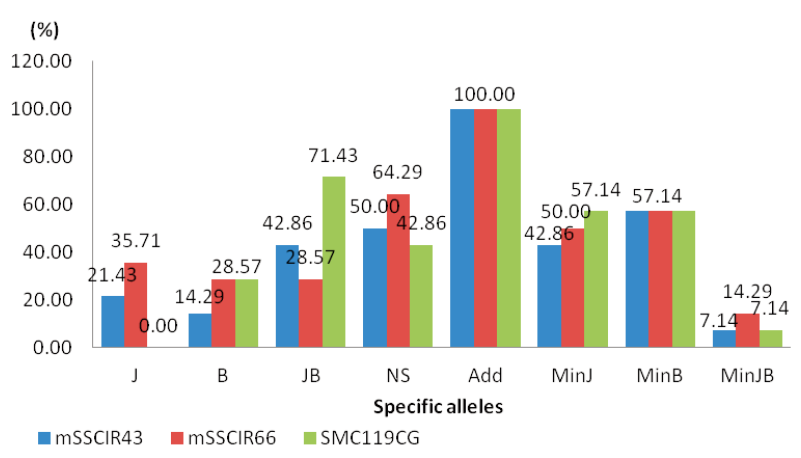

Figure 2. The ability of three primer to detect specific alleles of parents in F1. J: male specific alleles; B: female specific alleles; JB: male and female specific alleles present at different location; NS: male and female alleles present at the same location; Add: additional alleles; MinJ: eliminated male alleles; MinB: eliminated female alleles; MinJB: eliminated male \& female alleles

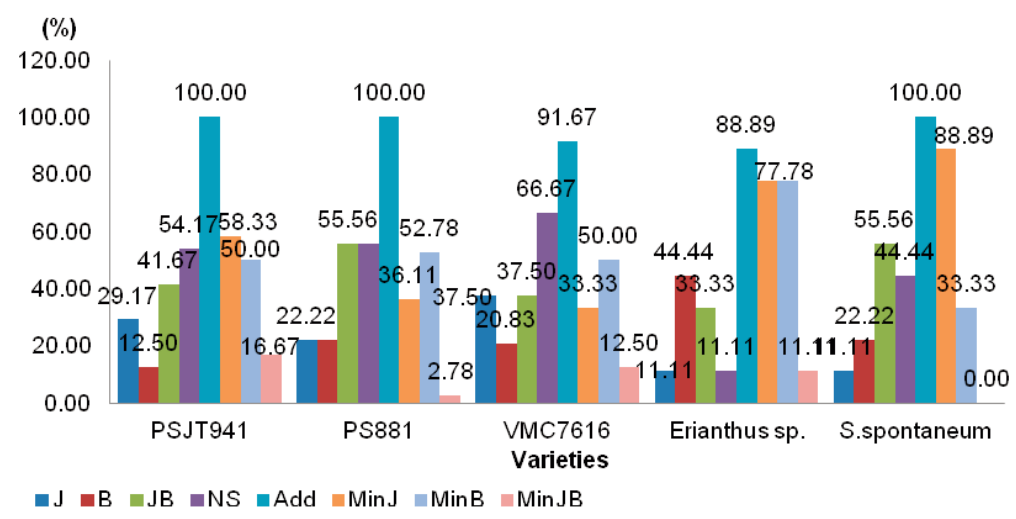

Figure 3. The average percentage of parent-specific alleles in F1. J: male specific alleles; B: female specific alleles; JB: male and female specific alleles presence at different location; NS: male and female alleles presence at the same location; Add: additional alleles; MinJ: eliminated male alleles; MinB: eliminated female alleles; MinJB: eliminated male \& female alleles 
bp) than the ability to detect male and female specific alleles in solitary. Moreover, the crosses also produced the new characteristic recombination indicated by the number of additional alleles that had been found (100\%) in F1 (Figure 1)

Figure 2 showed the ability of each primer to detect each specific allele of parents in F1. Each primer had different ability to detect the existences of parent specific alleles in each crosses except the ability to detect the axistences of additional alleles (Add), eliminated male (MinJ) and female alleles (MinB) which was relatively equal for all the primers.

SMC119CG less sensitive to detect the existences of male specific alleles (J) and common alleles (NS) but better $(71.43 \%)$ to detect male and female specific alleles in collectively (JB) than two others primer. Furthermore, SMC119CG also had relatively better ability to recognize female specific alleles (B) than mSSCIR43. mSSCIR66 less sensitive to detect male and female specific alleles in collectively (JB) but relatively better to identify male $(\mathrm{J})$ and female $(\mathrm{B})$ specific alleles, common alleles (JB) and eliminated

Table 1. Genetic marker of male and/or female that could be identified in F1 in three primers and their origin of the crosses

\begin{tabular}{|c|c|c|c|}
\hline \multirow[b]{2}{*}{ Parents } & mSSCIR43 & mSSCIR66 & SMC119CG \\
\hline & $\begin{array}{l}\text { Alleles location \& the origin of } \\
\text { crosses* }\end{array}$ & $\begin{array}{c}\text { Alleles location \& the origin of } \\
\text { crosses* }\end{array}$ & $\begin{array}{l}\text { Alleles location \& the origin of } \\
\text { crosses* }\end{array}$ \\
\hline PSJT941 & 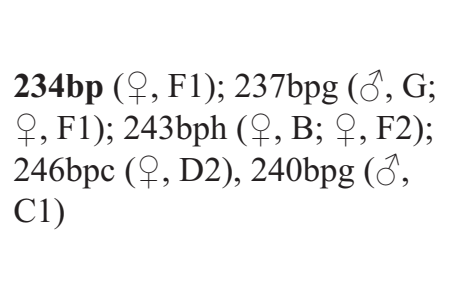 & 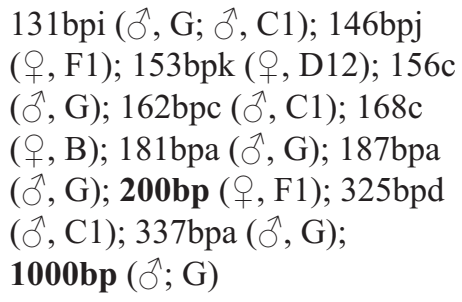 & 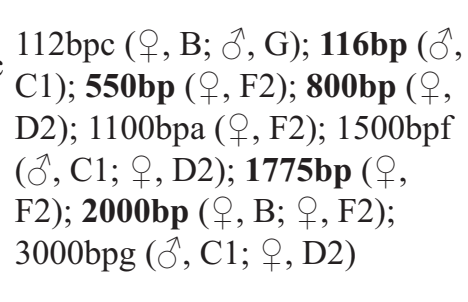 \\
\hline
\end{tabular}

\begin{tabular}{|c|c|c|}
\hline PS881 & 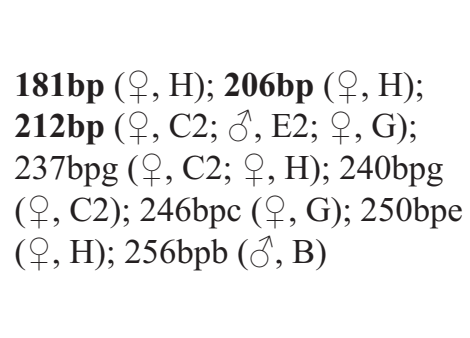 & 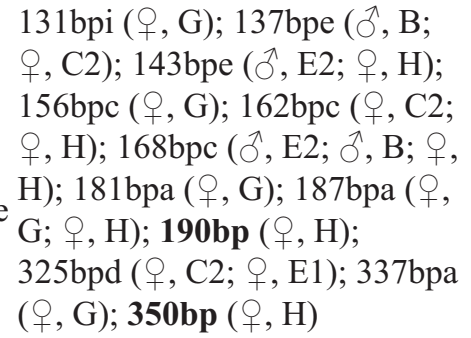 \\
\hline
\end{tabular}

131bpi (ㅇ, D1; + , C1); 137bpe ( (, $\mathrm{A} ;$;, E2); 143bpe $\left({ }^{\top}, \mathrm{H}\right)$; $237 \mathrm{bpg}(+\circ, \mathrm{H}) ; 240 \mathrm{bpg}(+, \mathrm{C} 1) ; 1$, $\mathrm{Abpk}\left(\hat{O}^{\hat{2}}, \mathrm{D} 2\right) ; 156 \mathrm{bpc}(+$,

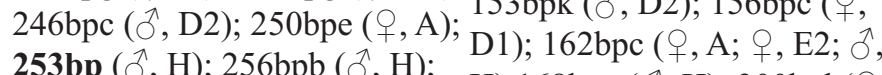

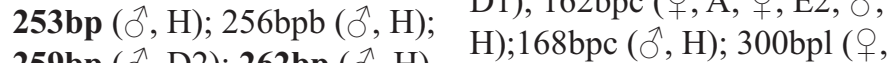

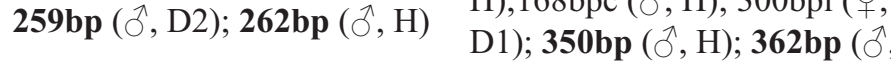
H) 106bp (つ̂, E2); 109bp (,$+ \mathrm{H})$; $112 \mathrm{bpc}(+, \mathrm{C} 2 ;$;, $\mathrm{G} ;$; $; \mathrm{H})$; $122 \mathrm{bp}(+, \mathrm{H}) ; 125 \mathrm{bpb}(+, \mathrm{H})$; $130 b p(O, H)$; 131 bp $(+, \mathrm{C} 2)$; $137 \mathrm{bpb}(q, \mathrm{E} 1 ;$;, $\mathrm{H}) ; \mathbf{1 4 0 b p}$ $(O, \mathrm{G}) ; 143 \mathrm{bpe}(+, \mathrm{C} 2 ; \hat{\jmath}, \mathrm{E} 2$;

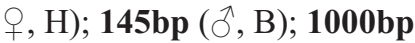

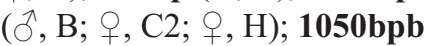
(ठ, E2); 1100bpa (ㅇ, H); 1150bp (う, E2); 1200bp (ふ, B; ㅇ, $\mathrm{C} 2 ;$ ㅇ, H); 3000bpg (ㅇ, E1) $112 \mathrm{bpc}\left(+, \mathrm{F} 2 ; \sigma^{\lambda}, \mathrm{H}\right) ; 120 \mathrm{bp}$ (,, $\mathrm{C} 1 ;$;, $\mathrm{D} 1 ; \AA, \mathrm{O} 2) ; 125 \mathrm{bpb}$ (ㅇ, A; ठ̀, H); 137bpb $(\hat{\jmath}, \mathrm{H})$; 143bpe (, C1;,$+ \mathrm{D} 1 ;$; , D2;

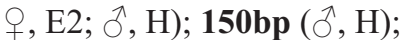
1625bp (ð, $\mathrm{H}) ; 1500 \mathrm{bpf}($, E2; $\widehat{\jmath}, \mathrm{H}) ; 3000 \mathrm{bpg}($ (, E2; तै $\mathrm{H})$

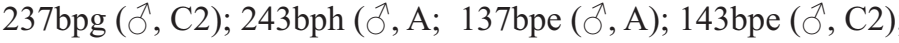

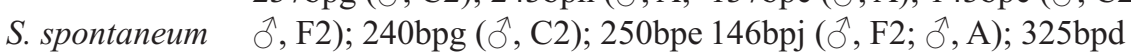
$(\stackrel{\jmath}{\mathrm{A})}$ $(\lesssim, A)$

\begin{tabular}{|c|c|c|}
\hline Erianthus sp. & $218 b p(\lesssim, F 1) ; 241 b p(\lesssim, D 1)$ & $\begin{array}{l}\text { 131bpi (ठ, E1; } \hat{O}, \mathrm{D} 1) ; 146 \mathrm{bpj} \\
(\hat{\jmath}, \mathrm{F} 1) ; 300 \mathrm{bpl}(\hat{\jmath}, \mathrm{D} 1)\end{array}$ \\
\hline
\end{tabular}

\footnotetext{
Note: * The origin of crosses; Bold: specific alleles; a: common alleles between PSJT941 \& PS881; b: common alleles between PS881 \& VMC7616; c: common alleles between PSJT941, PS881 \& VMC7616; d: common alleles between PSJT941, PS881 \& S.spontaneum; e: common alleles between PS881, VMC7616 \& S.spontaneum; f: common alleles between PSJT941, VMC7616 dan S.spontaneum; g: common alleles between PSJT941, PS881, VMC7616 \& S.spontaneum; h: common alleles between PSJT941 \& S.spontaneum; i: common alleles between PSJT941, PS881, VMC7616 \& Erianthus sp.; j: common alleles between PSJT941, S.spontaneum \& Erianthus sp.; k: common alleles between PSJT941 \& VMC7616; 1: common alleles between VMC7616 \& Erianthus sp.
} 
common alleles (MinJB) than two others primer.

Figure 3 showed the average percentation of parent specific alleles in F1. In crosses of Erianthus sp. and $S$. spontaneum as the parent, all primers could recognize the existances of female specific alleles (B) better than the crosess of PS881, PSJT941 and VMC7616 as the parent. Different result was found at the crosses of PSJT941 and VMC7616 as the parent. In this crossing, the primer could recognize the existences of male specific alleles $(\mathrm{J})$ better than female specific alleles (B). Different result also showed in crosses of PS881. In this crosses, all primers could recognize the existences of male $(\mathrm{J})$ and female $(\mathrm{B})$ specific equaly $(22.22 \%)$. Further, all primers had the average ability to identify the existences of male and female specific alleles in collectively (JB) and common alleles (NS) across all the crossing except in crosses of Erianthus sp. as the parent.

This study could also identify the eliminated alleles (the alleles that identified in parents but not identified in F1). Erianthus sp. and S. spontaneum had the most eliminated alleles than three others parent (sugarcane commercial varieties). The average of eliminated alleles detected at Erianthus sp. and $S$. spontaneum were $37.97 \%$ (95.38\%) dan $49.08 \%$ (123.29\%) better than three sugarcane commercial varieties in average, respectively. Erianthus sp. and $S$. spontaneum used as male parent in this study which is expected to improve various desirable characteristic, such as biotic and abiotic stress resistances. PSJT941 as the parent had nearly the same proportion of eliminated male and female alleles that were only $8,33 \%(16.66 \%)$ respectively. PS881 and VMC7616 as the parent had average eliminated female alleles $16.67 \%(46.16 \%)$ and $16.67 \%$ (50.01\%) bigger than average eliminated male alleles.

Table 1 listed the genetic marker of male and/or female parent that could be identified in F1. SMC119CG could detect more male and/or female genetic marker than mSSCIR43 and mSSCIR66. However, all primers had the same ability to detect male and/or female genetic marker at commercial sugarcane varieties. In PSTJ941, primers could detect one (mSSCIR43), two (mSSCIR66) and five (SMC119CG) male and/or female genetic marker. Moreover, in PS881, primers could detect three (mSSCIR43), two (mSSCIR66) and eleven (SMC119CG) male and/or female genetic marker, while in VMC7616, primer could detect three (mSSCIR43) and two (each mSSCIR66 and SMC119CG) male and/or female genetic markers.

Contrary with the commercial sugacane varieties, in crosses of Erianthus sp. or S. spontaneum as parent, only one to three male genetic putative marker detected by primers, fewer than commercial sugacane varieties. In $S$. spontaneum, only one primer (SMC119CG) could detect one male genetic marker, while two (mSSCIR43 and SMC119CG) and one (mSSCIR66) male genetic marker could be detected in Erianthus sp.

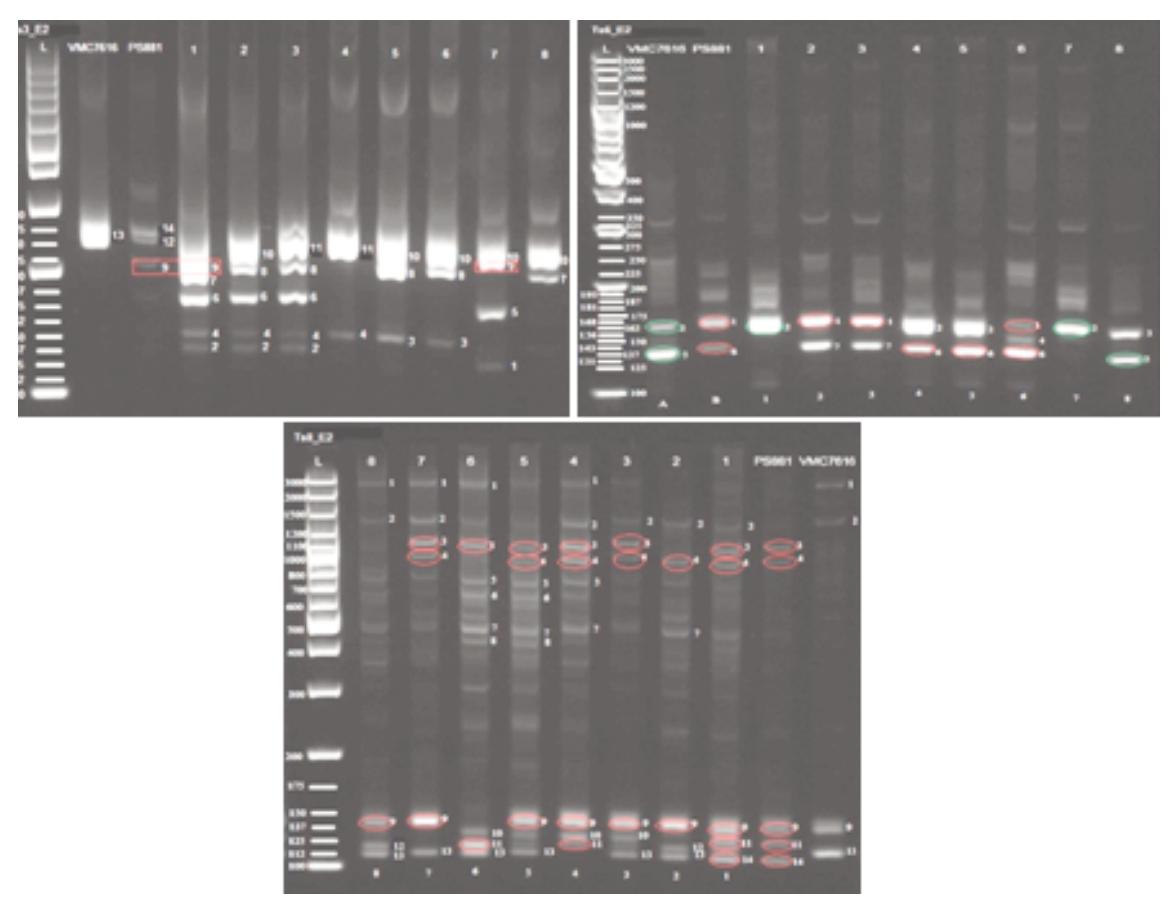

Figure 4. . Identification of male and female specific alleles in E2 crosses using three microsatellite primers. Left above: mSSCIR43 (Ts3), right above: mSSCIR66 (Ts6) and center bottom: SMC119CG (Ts8) 
There were 25 common alleles that could be identified. These common alleles presented at the same location among Saccharum spp. There were four allele between two sugarcane commercial varieties PSTJ941 and PS881, three alleles between two sugarcane commercial varieties PS881 and VMC7616, five allele between three sugarcane commercial varieties PSJT941, PS881 and VMC7616; one allele between two sugarcane commercial varieties PSJT941, PS881 and their wild relative $S$. spontaneum, three allele between two sugarcane commercial varieties PS881, VMC7616 and their wild relative $S$. spontaneum; one allele between two sugarcane commercial varieties PSJT941, VMC7616 and their wild relative $S$. spontaneum; three allele between three sugarcane commercial varieties PSJT941, PS881, VMC7616 and their wild relative S. spontaneum; one allelel between PSJT941 and S. spontaneum; one allelel between PSJT941, PS881, VMC7616 and their wild relatives Erianthus sp.; one allelel between PSJT941, S.spontaneum and Erianthus sp.; one allelel between PSJT941 and VMC7616 and one allele between VMC7616 dan Erianthus sp. (Table1). The existence of the common alleles among Saccharum spp and between Saccharum spp and Erianthus sp showed that there were genetic similarity among the parents.

Identification and distribution of male and female genetic marker at F1 (from the E2 crosses) was showed in Figure 4 and 5. All the alleles in mSSCIR43 that were identified in male and female parent presented

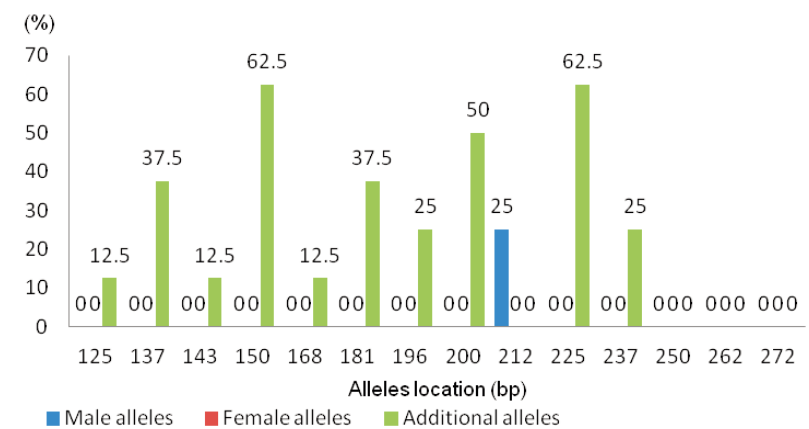

at different bp, therefore all the alleles could be used as male and female genetic marker. However, the female allele (262bp) and male alleles (250bp and 272bp) did not found in all of F1. It indicated that these alleles was eliminated. Only one male allele (212bp) could be identified in $\mathrm{F} 1$ with distribution value $25 \%$.

Furthermore, there were also no similarity of allele location between male and female parent detected by mSSCIR66, therefore all alleles that identified in both parents could be used as male and female genetic marker. In F1, two female alleles had distribution value $25 \%$ (137bp) and 50\% (162bp). While, two male alleles had the same distribution value $(37.5 \%)$.

SMC119CG could identify one allele (143bp) in both parents, therefore this allele could not be used as male and female genetic marker. Although in F1, this allele had the high distribution value (87.5\%). Three others allele could be used as male and female genetic marker. Distribution value of each alleles were $100 \%$ (112), 75\% (1500bp) and 50\% (3000bp) in female parent and 12,5\% (106bp) and 75\% (1050bp and 1150bp) in male parent.

These three primers could also identify the additional alleles in $\mathrm{F} 1$ but these alleles could not identify in parents. mSSCIR43 could identify ten additional alleles with distribution value $12.5-62.5 \%$, then mSSCIR66 could identify three additional alleles with distribution value $12.5-50 \%$ and SMC119CG could identify seven additional alleles with distribution value $25-50 \%$.
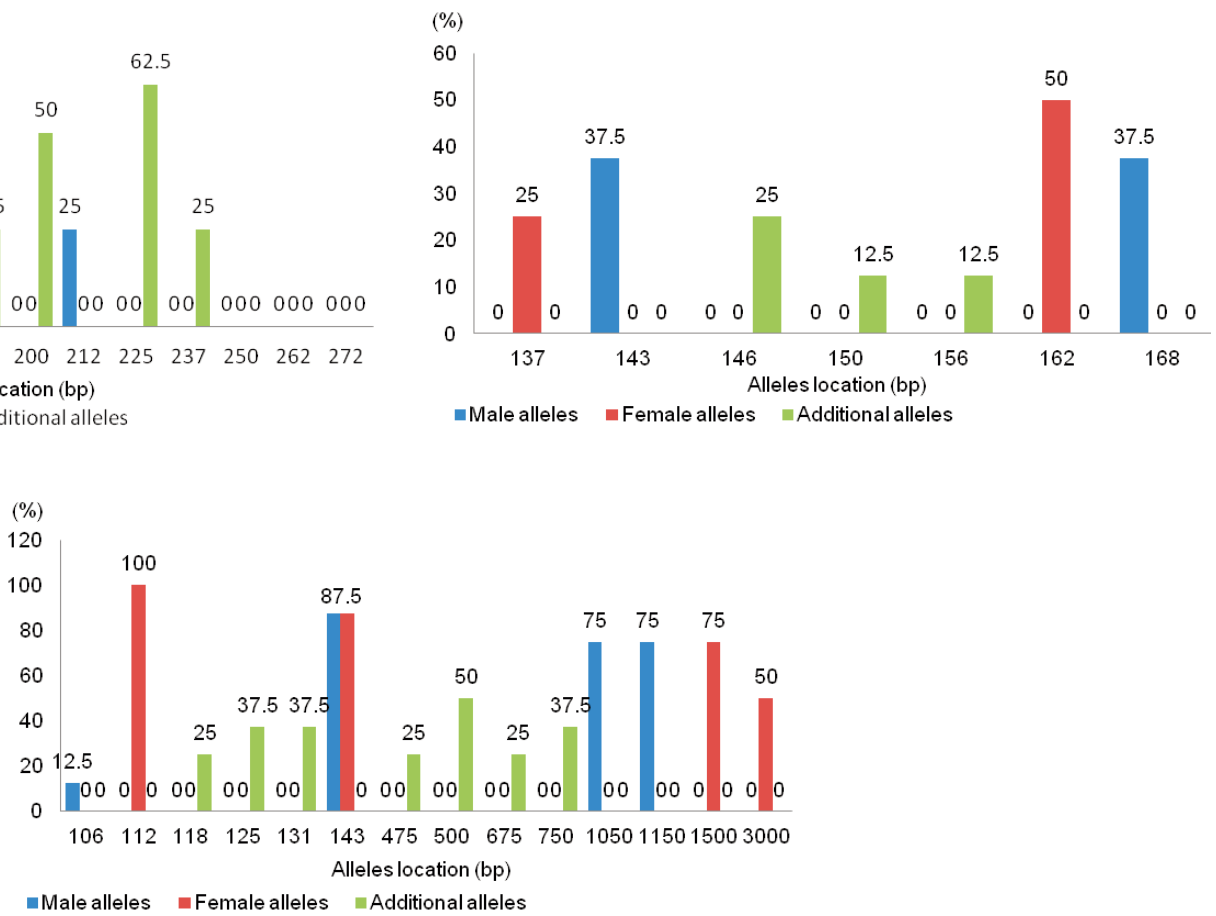

Figure 5. Distribution of male and female putative genetic marker at F1 (from the E2 crosses) using three microsatellite primers. Left above: mSSCIR43; right above: mSSCIR66; center bottom: SMC119CG 


\section{Discussion}

Attempts to cross Saccharum spp. with Erianthus sp. had limited success in view of the high genetic distance between the two genera and consequent low compatibility of such crosses. There is also difficulty in identifying the hybrids among the progenies that are obtained from such crosses. Since the major component of the hybrid genome is derived from the Saccharum parent which has a higher chromosome number, the progenies tend to be largely similar to Saccharum parent. Differentiate large number of selfed progenies arising from such crosses remains a difficult task. Molecular marker had been widely used in this context for identification of intra and intergeneric hybrids.

The first step should be done before identify the progenies is determining specific marker of parents (Figure1). The specific marker that has ability to identify the parents then used as male and female genetic marker in progenies (Figure 2). The study showed all the primers could identify $1-17$ specific alleles that were probably as male and female genetic marker with the average 9 alleles each parent from all crossing (Table 1). PS881 was the parent with the most genetic marker that could be identified (sixteen alleles) whereas S. spontaneum was the parent with the fewest genetic markers (two alleles). Furthermore, Erianthus sp. had three alleles, PSJT941 had eight alleles and VMC7616 had eleven alleles.

According to Govindaraj et al. (2012) using 15 microsatellite marker could identify the existence of 57, 53, 56 and 42 male specific alleles in putative hybrid that were resulted from Erianthus arundinaceus crosses. In addition, Santos et al. (2014) succeed identify 3-16 with the average 7.25 female specific alleles and 7-9 with the average 8.25 male specific alleles using three microsatellite marker at biparental crossing of commercial sugarcane varieties. Further, Costa et al. (2014) could identify seven polymorphic alleles in average using eight microsatellite marker both in parent and selfs progenies.

Using microsatellite marker, Chandra et al. (2014), succeed to identify 25 specific alleles of India sugarcane cultivar and 27 specific alleles of US sugarcane cultivar with 162 alleles were common to both India and US sugarcane cultivar. Another results, using RAPD marker, McIntery and Jackson (2001) succeed to identify 3-5 specific alleles of female parent and 5-8 specific alleles of male parent at the crossing between sugarcane commercial varieties. While 107 Erianthus-specific alleles in crossing of $S$. officinarum $\times$ E. ciliaris or S. officinarum $\times$ E. procerus could be identified by Nair et al. (2006) using microsatellite marker. Pan et al.
(2015) also could identify one female specific allele (HoCP 00-950), four male specific alleles (L 99-233) and one common allele.

There were 25 common alleles in Saccharum spp (Table 1). Nine alleles were identified between sugarcane commercial varieties and $S$. spontaneum, 13 alleles were identified among sugarcane commercial varieties, three alleles were identified between Saccharum spp and Erianthus sp. VMC7616 was the variety that had the most common alleles (20) while Erianthus sp. was the variety that had the fewest common alleles (three). Three others varieties had eigten (PSJT94), tweleve (VMC7616) and tweleve (S. spontaneum) common alleles.

These results were not surprising. Many study also reported that in many sugarcane crossing program around the world, the parents that used for crosses originated from inter- and intraspecific hybridization and its progenies. They results progenies with the same and share genetic makeup with parents (Stevenson, 1965; Zhang et al., 2001; Perera et al, 2012; Filho et al., 2010; Pan et al., 2003; Hapsoro et al., 2015; Chen et al., 2017). In contrast to previous result, in crosses between Erianthus sp. and sugarcane commercial varieties, there were only few common alleles that could be identified. This is likely due to the wide range of genetic distance between Erianthus sp. and sugarcane commercial varieties. The wide range of genetic distance between Erianthus sp. and Saccharum spp. probably become the limiting factor in crossing between Erianthus sp. and Saccharum spp. (Gao et al., 2015; Govindaraj et al., 2012).

Figure 4 and 5 were the example that illustrated male and female genetic markers and their existence in F1. Both figure illustrated male (PS881) and female (VMC7616) genetic markers and their existence in $\mathrm{F} 1$ in crosses E2. There were one to four female specific alleles and two to four male specific alleles could be identified by all primers but not all alleles presented in F1. One female specific alleles and two male-specific alleles were not present in F1. There was also one allele that presented in both parents (common alleles). Alongside with that, three to ten additional alleles could be identified in this crosses.

This study also found some eliminated alelles of parent (Figure 3). These alleles were identified in parents but were not found in progenies. Interestingly in crossing with PSJT941 as a male or female parent, there were equal value both of the elimination of male and female alleles. This indicated the utilization of PSJT941 as a parent will need more than one backcrossing generation to preserve the desirable characteristic 
of recipient (female parent) without lossing the desirable characteristic from donor (male parent) or need bridge crossing to add one or some desirable characteristic from donor (male parent) to recipient (female parent). The same result was found in Erianthus sp. However, in this study, Erianthus sp. only used as a male parent. This indicated in crossing between sugarcane as a female and Erianthus sp. as a male was a difficult thing to do. Beside that, with a lot of eliminated female parent alleles indicated only a few of progenies could produce or survive.

As parents, both PS881 and VMC7616 had more eliminated female parent alleles than male parent alleles. This indicated that when these varieties became as a female parents, both of PS881 and VMC7616 will need more than one backcrossing generation to maintain the desirable characteristic that had been existed. On the contrary, it seems more easier for PS881 and VMC7616 to write down one or some new desirable characteristics from donor to recipient parents (female parent).

The different result was found in S. spontaneum. As Erianthus sp., in this study $S$. spontaneum only used as male parent. It seems that in crossing with commercial sugarcane varieties as female parent, more male alleles will be eliminated. This indicated that it will be need more effort to insert the desire characteristic from $S$. spontaneum (donor, male parent) through to the sugarcane commercial varieties as the recipient parents (female parent). It also indicates progenies will have a lot of similar characteristics with female parent and there is a difficulty to distinguish the hybrid from the selfed

The events of parent allele elimination in progenies had been reported in previous study. In Erinathus sp. and $S$. spontaneum, losing of some alleles probably because of the wide genetic distance, incompatibility, steril hybrid or inconsintency of chromosome transmission pattern from parents to hybrid. This event will cause genomic imbalance. Nearby, the progenies from crossing have the similar characteristics with the female parent (S. officinarum) in which there is a difficulty to distinguish the progenies as a hybrid or selfed. Sugarcane is a polyploid plant with the higher amount and big genome size and also complex structure. In polyploid plant, during megasphorogenesis, unreduction gamete $(2 \mathrm{n})$ could yield unreduction ovule with 2n egg cell (Masom et al., 2015). The fusion of unreduction ovul and normal haploid pollen at fertilization are resulting $2 n+n$ transmission mechanism in sugarcane (Huang et al., 2015).

Ming et al. (1998) reported during meiotic phase, there were a lot of diploidization (preferential pairing) event at S. officinarum and S. robustum but only a little event occur at $S$. spontaneum. Diploidization (preferential pairing) has the important role to stabilize genetic transmission and it was the reflection of bivalent chromosome pairing mechanism (Sreenivasan et al., 1987). Congruent with the previous study, Janoo et al. (2004) also showed the same result. The chromosome transmission pattern at $S$. officinarum $\times S$. spontaneum, $S$. officinarum $\times$ commercial sugarcane variety and Saccharum spp. $\times$ Erinathus $\mathrm{sp}$. is $2 \mathrm{n}+\mathrm{n}$ until $\mathrm{BC} 1$ generation and from $\mathrm{BC} 2$ and the next generation the pattern become $\mathrm{n}+\mathrm{n}$ (Bremer, 1962; D'Hont et al., 1996, Nair et al., 2006; Aitken et al., 2007; Piperedis et al., 2010; Govindaraj et al., 2012; Nair et al, 2017).

Another study had been conducted by Wu et al. (2014), using genomic in situ hybridization (GISH) method there were unreguler chromosome transmission mechanism at the crossing of Saccharum spp. $\times E$. arundinaceus. The mechanisms are $\mathrm{n}+\mathrm{n}$ at $\mathrm{F} 1$ and $2 \mathrm{n}+\mathrm{n}$ at $\mathrm{BC} 1$. Both of this mechanism probably happen by nondijunction of homologous chromosomes (NHC) at the first meiotic division or nondijunction of sister chromatids (NSC) at the second meiotic division. Different result had been reported by Nair et al. (2017), that was showed the hybrid from crossing $E$. procerus $\times$ S. officinarum had $2 \mathrm{n}+\mathrm{n}$ chromosome transmission model in F1 with the elimination event in a small part of chromosome both of parents and $\mathrm{n}+\mathrm{n}$ chromosome transmission model in $\mathrm{BC} 1$. Beside that, the crosses could also increase the mean juice sucrose $\%$ in amount $90 \%$ higher than the $\mathrm{F} 1$ parents.

The eliminated chromosome event in hybrid had been reported by Piperidis and D'Hont (2001). Piperidis and D'Hont (2001) showed that there were one to nine eliminated chromosomes in hybrid from $S$. officinarum $\times$ E. arundinaceus crossing. Beside that, there was no hybrid with fully parents chromosome in $(n+n)$ mechanism and in a few event of intergenomic recombination. Wide genetic distance probably caused the rare event of intergenomic recombination.

Parent sequences that had beed eliminated in the progenies from polyploid plant crossing have the bigger frequencies than the ability to build the new sequence (Fu et al., 2016). Alongside with bivalent pairing, there were also univalent mechanism at meiotic division in sugarcane but in the rare frequent (Daniels and Roach, 1987) with as like as diploid plant model inheritance (Hogarth, 1987). Irregular meiotic division had also been reported by Grivet et al. (2001), Sigh et al. (2011), Perera et al. (2012), Filho et al. (2010). 
The ability to detect eliminated male and female specific alleles could be an indication of how many male and female alleles were eliminated in crosses that had been done. In plant breeding program, one aim of hybridization is to improve one or some characteristics. Beside the progenies has the desirable characteristic that has been existed, they are also expected to have one and some improved characteristics from donor. If the progenies has one or some improved characteristics but lose the good characteristic that had been existed, this indicate that there were elimination event during meiotic division. Backcrossing is needed to restore the good characteristic that has been loss while maintaining one or some improve characteristics from donor.

In sugarcane crossing there was a term of proven cross and proven parents. This term based on the appearance of progenies at the early stage of selection (Sukarso, 1986 in Budhisantoso, 2012). Therfore, the choice of parents and parents set is the crucial aspect in sugarcane breeding programe (Skinner, 1971 in Budhisantoso, 2012; Hapsoro et al., 2015; Chen et al., 2017). The choice of pricise parents and parents set will determine the success of the crossing by the appearance of progenies better than the average or the best parent value (heterosis).

In addition, there were also identified additional alleles that was not identified in parent but identified in progenies. The additional alleles that were different from both of parents showed the progeny from that crossing had the new genetic makeup in which different from the parents. The additional alleles results from segregation and fusion of parent gamete and resulting the new characteristics which play the important role to increase the genetic diversity in population. Sugarcane as a polyploid plant with the high amount and genome size, the different chromosome pairing at meiotic division could be happen and give the new genetic recombination in progeny.

D'Hont et al. (1996) discovered 10\% of progenies chromosome from crossing of R570 came from $S$. spontaneum and recombined with S. officinarum. This indicated there were only in a few the recombinations even in interspesific hydridization. The same results showed by Piperedis et al., (2010), that was 10-23\% of progenies chromosome originated from $S$. spontaneum and $8-13 \%$ was the result of interspecific recombination. Genetic recombination was also present in F1 crossing between Saccharum spp. and E. arundinaceus as reported by Huang et al. (2015). Huang et al. (2015) reported there were intergeneric chromosomal translocation resulted by genetic recombination in $\mathrm{BC} 1, \mathrm{BC} 2$ and $\mathrm{BC} 3$. This recombination inherited stably. The same result was reported by Pan et al. (2015). There were another alleles (non parental) that had been identified in $\mathrm{F} 1$ from crosses two sugarcane commercial varieties (HoCP 00-950 and L 99-233).

\section{CONCLUSIONS}

Microsatellite as one of the molecular markers could be the best choice in performing selection at the early stage of the crosses by identification of male and female genetic marker on each of the crosses. Beside that, this method could also identify the existence and elimination rate of male and female alleles and additional alleles based on their visualization in the progenies. Based on this study, all microsatellite molecular markers could identify Saccharum spp. and Erianthus sp. genetic marker in each crossing with different sensitifity for each primer : 2-11 specific alleles of Saccharum spp. and 1-2 specific alleles of Erianthus sp. While, 25 alleles identified as common alleles of Saccharum spp.: one allele presented in all Saccharum spp, one allele presented in all sugarcane commercial varieties and seven alleles presented between $S$. spontaneum and sugarcane commercial varieties. The male and female genetic markers were cleary observed in the progenies. These indicated there were allele transmissions from parents to progenies.

\section{ACKNOWLEDGEMENT}

We would like to acknowledge Indonesian Agency of Agricultural Research and Development (IAARD), Ministry of Agriculture Indonesia who provided master scholarship at Faculty of Agriculture, Gadjah Mada University. We thank also to Bambang Heliyanto, Ph.D., Dr. Sesanti Basuki and Mr. Abdurrahman from Indonesian Sweetener and Fiber Crops Research Institute, IAARD, who provided plant materials and laboratory facilities to conduct the research, Ms. Tantri Dyah Ayu Anggraeni for helping review the English grammar.

\section{REFERENCES}

Aitken, K., J. Li, L. Wang, C. Qing, Y.H. Fan and P. Jackson. 2007, Characterization of intergeneric hybrids of Erianthus rockii and Saccharum using molecular markers. Genetic Resources and Crop Evolution, 54, pp. 1395-1405.

Barbosa M.H.P., M.D.V. Resende, L.A.S. Dias and G.V.S. Barbosa. 2012. Genetic improvement of sugar cane for bioenergy: the Brazilian experience in network research with RIDESA. Crop Breed. Appl. Biotechnol, 12, 
pp. 87-98.

Bremer, G. 1961. Problem in breeding and cytology of sugarcane. Euphytica, 10, pp. 59-78.

Budhisantoso, H. 2012. The efficiency of parent selction and its cross combination on sugarcane breeding : a simulation approach. Dissertation. Gadjah Mada University. Yogyakarta. Indonesia.

Chandra, A., M.P. Grisham and Y.B. Pan. 2014. Allelic divergence and cultivar-specific SSR alleles revealed by capillary electrophoresis using fluorescence-labeled SSR markers in sugarcane. Genome, 57, pp. 363-372.

Chen, S, W.K. Shen, G.H. Xu, X.M. Wu, Q.Q. Deng and Z.M. Dou. 2017. Assessment of genetic relationship and diversity among Chinese sugarcane parental clones using Scot and ISSR markers. Int. J. Agric. Biol., 19 (2), pp. 291-298.

Costa, P.M.A., C.F. Almeida, G. Silveira, B. Soarez, D.C.F. Baffa, L.A. Peternelli, L.L. Bhering and M.H.P. Barbosa. 2014. Selfing confirmation in sugarcane by using simple sequence repeat markers: an individual reciprocal recurrent selection scheme. Genetics and Molecular Research, 13 (4), pp. 8962--8970.

Daniels, J. and Roach, B.T.. 1987. Taxonomy and Evolution. In: D.J. Heinz, ed., Sugarcane Improvement Through Breeding, Published by Elsevier. Amsterdam. Vol 1., pp. 7-84.

D'Hont, A., L. Grivet, P. Fieldmann, S. Rao, N. Berding and J. C. Glaszmann. 1996, Characterisation of the double genome structure of modern sugarcane cultivars (Saccharum spp.) by molecular cytogenetics. Molecular and General Genetics, 250, pp. 405-413.

Filho, L.S.C.D., P.P. Silva, J.M. Santos, G.V.S Barbosa, C.E. Ramalho-Neto, L. Soares, J.C.F. Andrade, C.Almeida. 2010. Genetic similarity among genotypes of sugarcane estimation by SSR and coefficient of parentage. Sugar Tech, 12(2), pp. 145-149.

Fu, D., A.S. Mason, M. Xiao and H. Yan. 2016. Effects of genome structure variation, homeologous genes and repetitive DNA on polyploidy crop research in the age of genomics. Plant Science, 242, pp. 37-46.

Gao, Y.J., X.H. Liu, R.H. Zhang, H. Zhou, J.X. Liao, W.X. Duan and G.M. Zhang. 2015. Verification of progeny from crosses between sugarcane (Saccharum spp.) and an intergeneric hybrid (Erianthus arundinaceus $\times$ Saccharum spontaneum) with molecular Markers. Sugar Tech, 17(1), pp. 31-35.

Govindaraj, P., A. Balamurugan and U.S. Natarajam. 2012. Indentification of intergeneric hybrid between Erianthus arundinaceus and Saccha- rum spontaneum through STMS markers. International Sugar Journal, 114(1361), pp. 350356.

Grivet L and P. Arruda. 2001. Sugarcane genomics : depicting the complex genome of an important tropical crop. Current Opinion in Plant Biology, 5, pp. 122-127.

Hapsoro, D., H.A. Warganegara, S.D. Utomo, N. Sriyani and Yusnita. 2015. Genetic diversity among sugarcane (Saccharum officinarum L) genotypes as shown by randomly amplified polymorphic DNA (RAPD). Agrivita, 37(2), pp. 247-257.

Hogarth, D.M. 1987. Genetic of sugarcane. In: D.J. Heinz, ed., Sugarcane improvement through breeding. Amsterdam : Elsevier, pp. 255-268

Huang, Y., J. Wu, P. Wang, Y. Lin, C. Fu., Z. Deng, Q. Wang, Q. Li, R. Chen and M. Zhang. 2015. Characterization of chromosome inheritance of the intergeneric BC2 and BC3 progeny between Saccharum spp and Erinathus arundinaceus. Plos One, 10(7), pp. 1-13.

Janoo, N., L. Grivet, J. David, A. D'Hont and J.C. Glaszmann. 2004. Differential chromosome pairing affinities at meiosis in polyploidy sugarcane revealed by molecular markers. Heredity, 93, pp. 460-467.

Lu, X., H. Zhou, Y.B. Pan, C.Y. Chen, J.R. Zhu, P.H. Chen, Y.R. Li, Q. Cai and R.K. Chen. 2015. Segregation analysis of mikrosatelite (SSR) marker in sugarcane polyploid. Genetics and Molecular Research, 14(4), pp. 18384-18395.

Masom, A.S. and J.C. Pires. 2015. Unreduced gametes : meiotic mishap or evolutionary mechanism? Trends in Genetics, 31(1), pp. 5-10.

McIntyre, C.L. and P.A. Jackson. 2001. Low level of selfing found in a sample of crosses in Australian sugarcane breeding programs. Euphytica, 117, pp. 245-249.

Melloni, M.L.G., M.S. Scarpari, L.R. Pinto, D. Perecin, M.A. Xavier and M.G.A. Landell. 2014. Selfing rate estimation in sugarcane under unfavorable natural conditions of crossing by using microsatellite markers. Genetics and Molecular Research, 13(1), pp. 2278-2289.

Ming, R., S.C. Liu, Y.R. Lin, J. da Silva, W. Wilson, D. Braga, A. van Deynze, T. F. Wenslaff, K.K. Wu, P.H. Moore, W. Burnquist, M.E. Sorrells, J.E. Irvine and A.H. Peterson. 1998. Detaild alignment of Saccharum and Sorghum chromosomes : comparative organization of closely related diploid and polyploidy genomes. Genetic, 150, pp. 1663-1682.

Nagarajan, R., S. Alarmelu, R.M. Shanti and S. Dharmodharan. 2001. A note on extent selfing 
in interspesific crosses of Sacharum. Sugar Tech, 3(4), pp. 180-181.

Nair, N.V., A. Selvi, T.V. Sreenivasan, K.N. Push palatha and S Mary. 2006. Characterization of intergeneric hybrids of Saccharum using molecular markers. Genet Resour Crop Evol, 53, pp. 163-169.

Nair, N.V., K. Mahonraj, K. Sunadaravelpandian, A. Suganya, A. Selvi and C. Appunu. 2017. Characterization of an intergeneric hybrid of Erianthus procerus $\times$ Saccharum officinarum and its backcross progenies. Euphytica, 213(267), pp. 1-13

Organisation for Economic Co-operation and Development (OECD). 2013. Consensus Document on the Biology of Sugarcane (Saccharum spp). No.56. Environmet Directorate. Organisation for Economic Co-operation and Development. Paris

Pan, Y.B. 2006. Highly polymorphic microsatellite DNA markers for sugarcane germplasm evaluation and variety identity testing. Sugar Tech, 8(4), pp. 246-256.

Pan, Y.B., G.M. Cordeiro, E.P. Richard Jr and R.J. Henry. 2003. Moleculer genotyping of sugarcane clones with microsatellite DNA markers. Maydica, 48, pp. 319-329.

Pan, Y.B., P. Liu dan Y.Que. 2015. Independent segregating simple sequence repeats (SSR) alleles in polyploidy sugarcane. Sugar Tech, 17(3), pp. 235-242.

Perera, M.F., M.E. Arias, D. Costilla, A.C. Luque, M.B. Garc1'a, C. Di'az Romero, J. Racedo, S. Ostengo, M.P. Filippone, M.I. Cuenya and A.P. Castagnaro. 2012. Genetic diversity assessment and genotype identification in sugarcane based on DNA markers and morphological traits. Euphytica, 185, pp. 491-510.

Piperidis, G and A. D'Hont. 2001. Chromosome composition Analysis of Various Saccharum intrspecific hybrids by Genonic In Situ Hibridizaton (GISH). Proc.Int.Soc.Sugar Cane Technol., 24, pp. 565-566.

Piperedis, G., N. Piperedis and A. D'Hont. 2010. Molecular cytogenetic investigation of chromosome composition and transmission in sugarcane. Mol Genet Genomic, 284, pp. 65-73.

Santos, J.M., G.V. de Souza Barbosa, C.E.R. Neto and C. Almeida. 2014. Efficiency of biparental crossing in sugarcane analyzed by SSR markers.
Crop Breeding and Applied Biotechnology, 14, pp. 102-107.

Singh, R.K., R.B. Singh, S.P. Singh, and M.L. Sharma. 2011. Identification of sugarcane microsatellites associated to sugar content in sugarcane and transferability to other cereal genomes. Euphytica, 182, pp. 335-354.

Sreenivasan, T.V., B.S. Ahloowalia and D.J. Heinz. 1987. Cytogenetics. In: D.J. Heinz, ed., Sugarcane Improvement Through Breeding.(). Amsterdam: Elsevier, , pp. 211-253.

Stevenson, G.C. 1965. Genetics and Breeding of Sugarcane. London: Longmans, Green, , UK. pp. 284

Tew, T.L. and Y.B. Pan. 2010. Microsatelite (simple sequence repeat) marker-based paternity analysis of a seven-parent sugarcane polycross. Crop Sci, 50, pp. 1401-1408.

Wang, X.H., Q.H. Yang, F.S. Li, L.L. He and S.C. He. 2009. Molecular indentification of Saccharum spp. $\times$ Erinathus fulvus hybrids using sequence characterized amplified region markers. Crop Sci., 49, pp. 864-870.

Wu, J., Y. Huang, Y. Lin, C. Fu, S. Liu, Z. Deng, Q. Li, Z. Huang, R. Chen and M . Zhang. 2014. Unexpected inheritance pattern of Erianthus arundinaceus chromosomes in the intergeneric progeny between Saccharum spp and Erianthus arundinaceus. Plos One, 9(10), pp. 1-9.

Xavier, M.A., L.R. Pinto, T.M. Favero, D. Perecin, L.A. Carlini-Garcia and M.G.A. Landell. 2014. Paternity indentification in sugarcane polycross by using microsatelite markers. Genetics and Molecular Research, 13(1), pp. 2268-2277.

You, Q., L. Xu, Y. Zheng and Y. Que. 2013. Genetic diversity analysis of sugarcane parent in chinese breeding programmes using SSR markers. Hindawi Publishing Corporation. The Scientific World Journal, 2013, pp. 1-11. Zhang, M.Q., A.L. Yu And R.K. Chen. 2001. Utility of SSRs for determining genetic similarities and relationships In Saccharum and its related Genera. Proc. Int. Soc. Sugar Cane Technol., 24, pp. 630-631. 